\title{
ESTUDO DO BALANCEAMENTO ESTEQUIOMÉTRICO PARA OTIMIZAÇÃO DE ARGAMASSAS DE REVESTIMENTO *
}

\section{Resumo}

Afonso Garcez de Azevedo ${ }^{1}$ Sérgio Neves Monteiro ${ }^{2}$ Jheison Lopes dos Santos ${ }^{3}$

As primeiras argamassas utilizadas em construções pelo homem continham em sua composição, materiais argilosos. Porém, por ser um material com muitas cargas elétricas livres, as argilas reagem constantemente com a água, ocasionando grande variação linear que acaba causando fissuração nas argamassas feitas com esse material. Assim, para solucionar esse problema, passou-se a utilizar cal hidratada no lugar dos materiais argilosos. Dessa maneira, mesmo não sendo composta por argila, a argamassa mantém sua trabalhabilidade. Nesse cenário, o presente trabalho teve como objetivo balancear estequiometricamente a cal hidratada e as argilas estudadas, para utilizar essas quantidades em argamassas para revestimento, barateando assim o material. Para encontrar esse balanceamento estequiométrico calculou-se a massa molar para um mol de cal hidratada e para um mol teórico de argila. Assim foram estipuladas algumas proporções possíveis de balanceamento da equação de reação cal+argila. Além disso, foram confeccionados corpos de prova, variando as quantidades de argila e cal, para realização de ensaios de capilaridade, a fim de comprovar a eficácia dos resultados encontrados no balanceamento teórico.

Palavras-chave: Argamassas; Revestimento; Estequiometria.

\section{STUDY OF STOICHIOMETRIC BALANCING FOR OPTIMIZATION OF COATING} MORTARS

\section{Abstract}

The first mortars used in man-made constructions contained in their composition, clay materials. However, since it is a material with many free electric charges, the clays react constantly with water, causing great linear variation that ends up causing cracking in the mortars made with this material. Thus, in order to solve this problem, hydrated lime was used instead of clay materials. In this way, even though it is not composed of clay, the mortar maintains its workability. In this scenario, the present work had as objective to balance stoichiometrically the hydrated lime and the clays studied, to use these amounts in mortars for coating, thus reducing cost of the material. To find this stoichiometric equilibrium, the molar mass was calculated for one mole of hydrated lime and one theoretical mole of clay. Therefore, some possible proportions of the equilibrium reaction of the lime + clay reaction were stipulated. In addition, samples were prepared by varying the amounts of clay and lime to perform capillary tests, in order to prove the efficacy of the results found in theoretical balancing.

Keywords: Mortars; Coatings; Stoichiometry

1 Doutorando em Engenharia Civil, Departamento de Engenharia Civil, Universidade Estadual do Norte Fluminense, Campos dos Goytacazes, RJ, Brasil.

2 Ph.D. em Engenharia de Materiais, Professor, Seção de Engenharia Mecânica e de Materiais, Instituto Militar de Engenharia, Rio de Janeiro, RJ, Brasil

3 Doutor em Ciência dos Materiais, Pós-Doutorando, Seção de Engenharia Mecânica e de Materiais, Instituto Militar de Engenharia, Rio de Janeiro, RJ, Brasil. 


\section{INTRODUÇÃO}

Há evidências de que as argamassas de revestimento mais antigas produzidas pelo homem eram feitas a partir de argila, gesso e outros aglomerantes [1]. Diversas obras históricas, como a pirâmide de Quéops no Egito e o Coliseu em Roma, presentes nas figuras 1 e 2 respectivamente, foram construídas com esse tipo de argamassa [1-3]. Destaca-se que essas obras mantêm parte de sua estrutura original até os dias de hoje, milhares de anos após suas construções.

O uso do material argiloso propiciava uma das propriedades mais importantes para as argamassas: a trabalhabilidade. Porém o aparecimento de patologias nas argamassas produzidas com esse tipo de material, fez com que ele caísse em desuso. Tais manifestações patológicas apareciam por causa das cargas livres presentes na argila, que absorvem e eliminam água com muita facilidade. Assim a argamassa expandia e retraia constantemente, sempre que absorvesse água presente no solo por capilaridade, o que causava danos à estrutura da argamassa, que deixava de cumprir sua função de proteção à alvenaria [4-5].

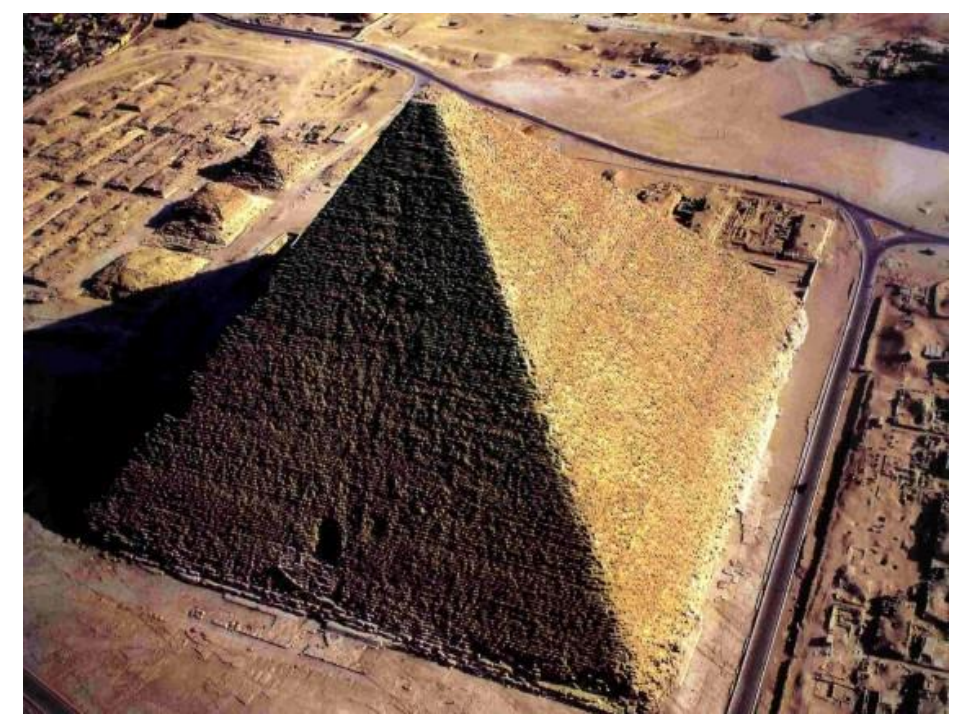

Figura 1. Pirâmide de Quéops [6].

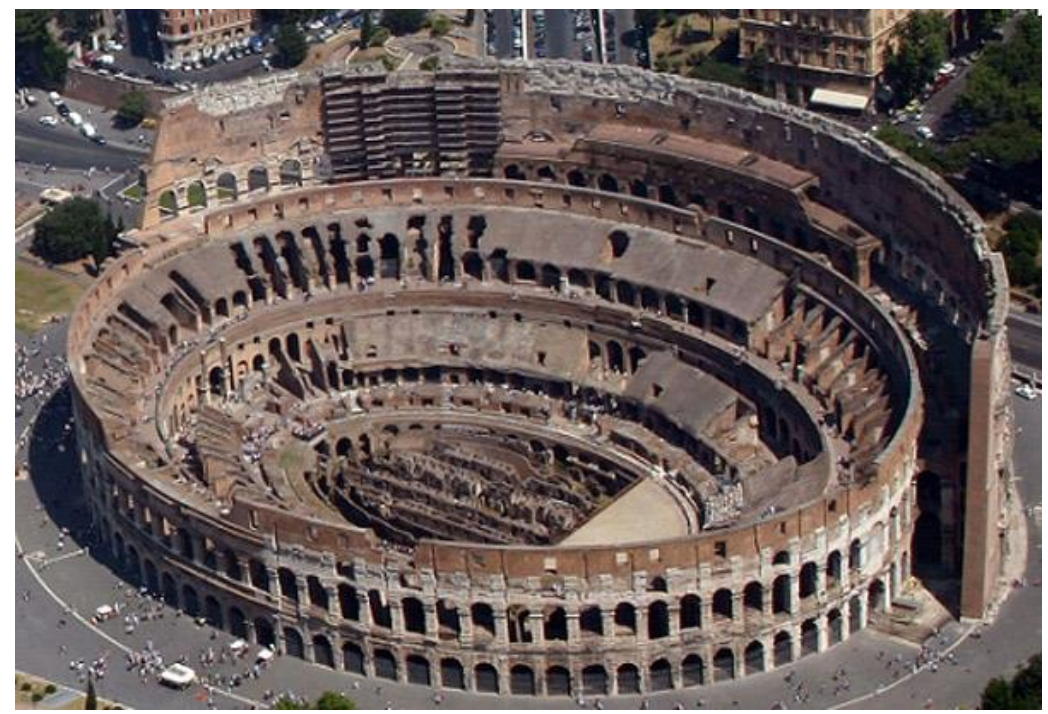

Figura 2. Coliseu de Roma [7]. 
Uma solução para esse problema foi substituir a argila por outro material que também conferisse trabalhabilidade a argamassa. Assim surgiram as argamassas confeccionadas com cal hidratada. Hoje em dia, praticamente todas as argamassas de múltiplo uso empregadas em construção civil são fabricadas com cimento, cal hidratada e areia.

O presente artigo pretende estudar uma maneira de balancear as cargas elétricas presentes na argila com o uso de cal hidratada. Dessa maneira seria empregada uma mistura de cal hidratada e argila, que além de conferir excelente trabalhabilidade as argamassas, não apresentariam problemas patológicos. Destaca-se também que o uso de argila em substituição a cal hidratada, ou o uso conjunto dos dois, é um importante avanço ao setor da construção civil, já que o material argila é muito mais barato do que a cal hidratada. Portanto essa nova técnica proporcionaria economias significativas ao sistema revestimento.

Tabela 2. Classificação de RCC de acordo com as resoluções 307 e 308 do CONAMA.

\begin{tabular}{cc}
\hline Classificação & Tipologia \\
\hline Classe A & $\begin{array}{c}\text { Resíduos reutilizáveis ou recicláveis como agregados, } \\
\text { tais como: a) obras de construção, demolição, } \\
\text { renovação e pavimentação, e outros trabalhos de } \\
\text { infraestrutura, incluindo o solo proveniente de } \\
\text { escavações; b) obras de construção, demolição, } \\
\text { renovação e reparação de edifícios: componentes } \\
\text { cerâmicos (tijolos, blocos, ladrilhos, tábuas, etc.), } \\
\text { argamassa e concreto; c) processo de fabricação e/ou } \\
\text { demolição de peças de concreto pré-moldado (blocos, } \\
\text { tubos, bordos, etc.) produzidos em locais de } \\
\text { construção; }\end{array}$ \\
\hline Classe B & $\begin{array}{c}\text { Resíduos recicláveis para outras utilizações, como: } \\
\text { plásticos, papel, metal, vidro, madeira e outros; }\end{array}$ \\
\hline Classe C & Resíduos para os quais as tecnologias ou aplicações \\
& economicamente viáveis não são projetadas para \\
permitir sua reciclagem/recuperação, como produtos a & partir do gesso; \\
\hline Classe D & Resíduos perigosos do processo de construção, como \\
& amianto, tintas, solventes, óleos e outros \\
& contaminados, ou aqueles provenientes de \\
& demolições, renovações e reparos de clínicas \\
radiológicas, instalações industriais e outros;
\end{tabular}

\section{MATERIAIS E MÉTODOS}

\subsection{Materiais utilizados}

Nesse artigo foram utilizados os seguintes materiais: cimento portland CP II-E-32 do fabricante Mauá, cal hidratada Super Cal $\mathrm{CH}$ III, areia lavada de rio e material argiloso proveniente de jazidas do município de Campos dos Goytacazes.

O material argiloso foi tratado antes de sua aplicação nas argamassas. $O$ tratamento do material incluiu as etapas de secagem natural, destorroamento, secagem em estufa e peneiramento na peneira \#200. 


\subsection{Dosagem dos traços}

O traço padrão utilizado, em massa, foi 1:1:6, que representa as proporções 1 de cimento, 1 de cal hidratada e 6 de areia. Esse traço foi escolhido pelo seu uso ser consagrado no estudo de argamassas de revestimento, já tendo sido usado por vários autores [8-15]. Assim, o primeiro traço a ser definido foi o que não teria nenhuma incorporação de material argiloso, representando o traço $0 \%$. Outro traço interessante a ser estudado é aquele que contém apenas incorporação de argila, sem nenhum teor de cal hidratada, sendo dessa maneira o traço $100 \%$.

Os demais traços foram obtidos através do balanceamento estequiométrico da reação teórica entre cal hidratada e a argila estuda. A Tabela 1, apresentada a seguir, especifica as massas atômicas utilizadas para cálculo da massa molar da cal hidratada e da argila teórica.

Tabela 1. Massa Atômica de alguns elementos químicos.

\begin{tabular}{cc}
\hline $\begin{array}{c}\text { Elemento } \\
\text { Químico }\end{array}$ & Massa Atômica (g) \\
\hline $\mathrm{H}$ & 1,00 \\
\hline $\mathrm{O}$ & 16,00 \\
\hline $\mathrm{Al}$ & 26,98 \\
\hline $\mathrm{Si}$ & 28,08 \\
\hline $\mathrm{Ca}$ & 40,08
\end{tabular}

Assim, sabendo que a fórmula da cal hidratada é $\mathrm{Ca}(\mathrm{OH})_{2}$ e que a da argila teórica é $\mathrm{Al}_{2} \mathrm{O}_{3} .2 \mathrm{SiO}_{2} . \mathrm{H}_{2} \mathrm{O}$, é possível calcular as massas molares desses dois compostos. Os valores de massa molar encontrados foram $74,08 \mathrm{~g} / \mathrm{mol}$ e $240,12 \mathrm{~g} / \mathrm{mol}$ para a cal hidratada e a argila, respectivamente. Sabendo que a reação entre esses dois compostos se processa da maneira apresentada na Equação 1, é possível encontrar novos traços arbitrando valores para $x$ e $y$, que representam as quantidades de mol de cada componente a serem reagidos. Os traços estudados nesse trabalho são apresentados na Tabela 2 .

$$
\mathrm{x} \cdot \mathrm{Ca}(\mathrm{OH})_{2}+\mathrm{y} \cdot \mathrm{Al}_{2} \mathrm{O}_{3} \cdot 2 \mathrm{SiO}_{2} \cdot \mathrm{H}_{2} \mathrm{O} \rightarrow \text { PRODUTO }
$$

Tabela 2. Traços intermediários com balanceamento x:y.

\begin{tabular}{cccccc}
\hline $\begin{array}{c}\text { Traço } \\
\text { (x:y) }\end{array}$ & $\begin{array}{c}\text { Mols de Cal } \\
\text { Hidratada (x) }\end{array}$ & $\begin{array}{c}\text { Massa de } \\
\text { Cal } \\
\text { Hidratada (g) }\end{array}$ & $\begin{array}{c}\text { Mols de } \\
\text { Argila } \\
\text { Hidratada (x) }\end{array}$ & $\begin{array}{c}\text { Massa de } \\
\text { Argila } \\
\text { Hidratada (g) }\end{array}$ & $\begin{array}{c}\text { Porcentagem } \\
\text { de Argila }\end{array}$ \\
\hline $1: 1$ & 1 & 74,08 & 1 & 240,12 & $76 \%$ \\
\hline $1: 2$ & 1 & 74,08 & 2 & 480,24 & $87 \%$ \\
\hline $2: 1$ & 2 & 148,16 & 1 & 240,12 & $62 \%$ \\
\hline
\end{tabular}

Para determinação da quantidade de água a ser utilizada em cada traço foi realizado o ensaio de consistência que segue a NBR 13276 Brazilian standard [16]. Nesse ensaio certa quantidade padrão de cada traço de argamassa é colocada, ainda no estado fresco, em um molde de tronco de cone em cima de uma mesa de consistência. Destaca-se que a quantidade de água acrescentada num primeiro momento é arbitrada. Após seguir o procedimento descrito na referida norma para aplicação da argamassa no molde, esse é retirado e a mesa inicia seus movimentos de descida e subida, para assim provocar o espalhamento da argamassa. Esse 
movimento é repetido 30 vezes. Após a última movimentação, o espalhamento da argamassa é medido com uma régua em três pontos diferentes.

Se a média do espalhamento obtido for de $(260 \pm 5) \mathrm{mm}$, admite-se que a quantidade de água arbitrada nessa primeira tentativa é adequada para que a argamassa possua trabalhabilidade adequada. Caso o espalhamento seja superior ao valor préestipulado na norma, o ensaio deve ser repetido diminuindo a quantidade de água já que o traço apresentou fluidez excessiva. Caso o espalhamento seja inferior a $(260 \pm 5) \mathrm{mm}$, o ensaio deve ser repetido com maior quantidade de água, porque o pequeno espalhamento indica que a argamassa não está trabalhável o bastante. 0 ensaio deve ser repetido até que se obtenha um espalhamento compatível com o normatizado na NBR 13276 Brazilian standard [16].

\subsection{Moldagem dos Corpos de Prova}

Para execução do ensaio de absorção e água por capilaridade, os corpos de prova foram moldados conforme metodologia da NBR 13279 Brazilian standard [17]. A argamassa foi misturada em um misturado padrão, onde se colocam os materiais em suas devidas proporções e a água na quantidade obtida no ensaio de consistência. Após homogeneizar os componentes da argamassa, e deixá-los descansar por 15 minutos conforme estipula a norma, a argamassa ainda no estado fresco foi aplicada em moldes prismáticos de $4 \mathrm{~cm} \times 4 \mathrm{~cm} \times 16 \mathrm{~cm}$. Após a aplicação, o material recebeu vibração com auxílio da mesa de consistência para que os corpos de prova ficassem bem compactos. Após uma semana, os corpos de prova foram desformados, e ficaram em processo de cura até atingir a idade de 28 dias, quando então foram ensaiados. Para cada traço estudo, 3 corpos de provam foram confeccionados.

\subsection{Ensaio de Absorção por capilaridade}

Após 28 dias de cura, os corpos de prova foram submetidos ao ensaio de absorção de água por capilaridade, que segue as prescrições da NBR 15259 Brazilian standard [18].

Para execução dos ensaios, as superfícies dos corpos de prova foram lixadas com lixa grossa. Em seguida, determinou-se a massa inicial $(\mathrm{m} 0)$, em gramas, para cada amostra. Essas foram posicionadas com a face quadrada lixada sobre suportes porosos no recipiente de ensaio, evitando a molhagem das outras superfícies. $O$ nível da água para o ensaio foi constante e igual a $(5 \pm 1) \mathrm{mm}$ acima da face em contato com a água.

A partir da colocação das amostras, foram determinadas as massas, em gramas, aos 10 minutos (m10) e aos 90 minutos (m90) para cada corpo de prova. Antes da pesagem, a superfície das amostras foram previamente enxutos com um pano úmido para evitar que as gotículas de água contribuíssem com o peso do corpo de prova.

Depois de realizado o ensaio é possível obter um parâmetro muito importante: o coeficiente de capilaridade $(\mathrm{C})$, definido pela norma como o coeficiente angular da reta que passa pelos pontos representativos das determinações realizadas aos 10 minutos e 90 minutos. Na prática para calcular o coeficiente, basta usar a Equação 2 apresentada a seguir, com as massas em g:

$$
C=\left(m_{90}-m_{10}\right)
$$


Pela norma brasileira, o resultado do coeficiente de capilaridade é dado em $\mathrm{g} / \mathrm{dm}^{2} \mathrm{xmin} \mathrm{m}^{1 / 2}$. Assim fica relativamente fácil usar esse parâmetro para comparar 0 comportamento em relação à capilaridade da argamassa estudada com outros trabalhos brasileiros, como os trabalhos das referências [13] e [19]. Porém fica mais difícil fazer a mesma análise com trabalhos internacionais, uma vez que estes usam um procedimento diferente da ABNT.

Para comparar com o trabalho [14] e [15] é necessário lançar mão da norma espanhola UNE EN 1015-18 [20], que apresenta metodologia de ensaio similar a norma brasileira, utilizando corpos cilíndricos com $4 \mathrm{~cm} \times 4 \mathrm{~cm} \times 16 \mathrm{~cm}$ e realizando a anotação das massas aos 10 e 90 minutos. A diferença é que o coeficiente de capilaridade por essa norma é dado em $\mathrm{kg} / \mathrm{m}^{2} \cdot \mathrm{min}^{1 / 2}$ e portanto é necessário realizar uma conversão do valor calculado conforme norma brasileira [18].

\section{RESULTADOS E DISCUSSÃO}

Para realizar a análise da absorção de água por capilaridade, parâmetro importante para entender se o balanço estequiométrico teve êxito ou não, inicialmente foi preciso definir a quantidade ideal de água para cada traço seguindo o procedimento da NBR 13276 Brazilian standard [18]. A tabela 3, indicada a seguir, mostra a quantidade ideal de água para cada traço e seu correspondente valor de espalhamento obtido através do procedimento descrito em Materiais e Métodos. É importante frisar que os valores de água obtidos no ensaio devem ser respeitados, para que a trabalhabilidade das argamassas não sofra nenhuma alteração e respeite as faixas estipuladas pela norma.

Tabela 3. Traços intermediários com balanceamento x:y.

\begin{tabular}{ccccccc}
\hline Traço & $\begin{array}{c}\text { Cimento } \\
(\mathbf{g})\end{array}$ & Argila $(\mathbf{g})$ & Cal $\mathbf{( g )}$ & Areia $(\mathbf{g})$ & $\begin{array}{c}\text { Água } \\
\mathbf{( g )}\end{array}$ & $\begin{array}{c}\text { Espalhamento } \\
\text { médio }(\mathbf{m m})\end{array}$ \\
\hline $\mathrm{A} 0 \%$ & 150 & 0 & 150 & 900 & 240 & 261,6 \\
\hline $\mathrm{A} 62 \%$ & 150 & 93 & 57 & 900 & 218 & 261,33 \\
\hline $\mathrm{A} 76 \%$ & 150 & 114 & 36 & 900 & 215 & 261,77 \\
\hline $\mathrm{A} 87 \%$ & 150 & 130,5 & 19,5 & 900 & 212 & 260 \\
\hline $\mathrm{A} 100 \%$ & 150 & 150 & 0 & 900 & 210 & 259 \\
\hline
\end{tabular}

Os resultados obtidos no ensaio de absorção de água por capilaridade para cada um dos traços estudados são apresentados na Tabela 4. Destaca-se que para uma correta interpretação dos resultados é importante realizar o ensaio respeitando o tempo de cura correto (28 dias), e seguindo o procedimento já descrito em Materiais e Métodos. Os valores estão indicados em $\mathrm{g} / \mathrm{dm}^{2} \times \mathrm{min}^{1 / 2}$ para comparação com os trabalhos [12] e [19] e em kg/m². $\mathrm{min}^{1 / 2}$ para comparação com os trabalhos [13-15].

Tabela 4. Coeficientes de Capilaridade obtidos para cada traço.

\begin{tabular}{ccc}
\hline Traço & $\mathbf{g} / \mathbf{d m}^{2} \mathbf{x} \mathbf{m i n}^{\mathbf{1} / \mathbf{2}}$ & $\mathbf{~} \mathbf{g} / \mathbf{m}^{\mathbf{2}} . \mathbf{m i n}^{\mathbf{1} / \mathbf{2}}$ \\
\hline $\mathrm{A} 0 \%$ & 9,67 & 0,967 \\
\hline $\mathrm{A} 62 \%$ & 11,07 & 1,107 \\
\hline $\mathrm{A} 76 \%$ & 11,67 & 1,167 \\
\hline $\mathrm{A} 87 \%$ & 11,53 & 1,153 \\
\hline $\mathrm{A} 100 \%$ & 12,50 & 1,250 \\
\hline
\end{tabular}

A Tabela 5 indica os valores de coeficiente de capilaridade encontrados por [13], que utilizou em seus estudos três tipos de argamassa 1:1:6 (A61, A62 e A63) variando a 
granulometria dos agregados de uma mistura para a outra. Nessa mesma tabela estão inclusos os valores encontrados por [19], que não utilizou argamassas confeccionadas em laboratório com traços 1:1:6, mas sim argamassas industrializadas no Brasil em seus estudos ( $A 1, A 2, A 3, A 4, A 7, A 12, A 16, A 17)$. Ambos autores [13] e [19] se basearam na norma brasileira [18] e por isso os valores de coeficientes estão em $\mathrm{g} / \mathrm{dm}^{2} \times \mathrm{min}^{1 / 2}$.

Tabela 5. Coeficientes de Capilaridade $[13,19]$.

\begin{tabular}{|c|c|}
\hline Traço & $\mathrm{g} / \mathrm{dm}^{2} \mathrm{xmin} \mathrm{min}^{1 / 2}$ \\
\hline $\mathrm{A} 0 \%$ & 15,67 \\
\hline A61 [13] & 18,64 \\
\hline A62 [13] & 14,91 \\
\hline A63 [13] & 9,00 \\
\hline A1 [19] & 8,70 \\
\hline A2 [19] & 8,70 \\
\hline A3 [19] & 10,40 \\
\hline A4 [19] & 12,10 \\
\hline A7 [19] & 9,70 \\
\hline A12 [19] & 11,30 \\
\hline A16 [19] & 13,20 \\
\hline A17 [19] & 15,67 \\
\hline
\end{tabular}

A Tabela 6 apresenta os valores de coeficientes de capilaridade das argamassas estudadas por [12] no traço 1:1:6 (REF, REFP25, REFC, FC15, FC30, A50, P50, 025, P25FC30, P25FPP, V50, V25), sempre mantendo constante as quantidades de cimento e cal hidratada e utilizando diferentes tipos de agregados. Nessa mesma tabela estão indicados os valores encontrados por [13] para argamassas no traço 1:1:6 utilizando agregado de concreto reciclado (recycled concrete aggregate) em substituição ao agregado natural (natural aggregate) em diferentes proporções (CLM0\%, CLM25\%, CLM50\%, CLM100\%). Destaca-se que os valores estão em $\mathrm{kg} / \mathrm{m}^{2} \cdot \mathrm{min}^{1 / 2}$ por atentarem para a norma espanhola [20].

Tabela 6. Coeficientes de Capilaridade $[14,15]$.

\begin{tabular}{cc}
\hline Argamassa & $\mathbf{~ k g} / \mathbf{m}^{\mathbf{2}} \mathbf{.} \mathbf{m i n}^{\mathbf{1} / \mathbf{2}}$ \\
\hline REF [14] & 1,30 \\
\hline REFP25 [14] & 1,65 \\
\hline REFC [14] & 0,53 \\
\hline FC 15 [14] & 0,65 \\
\hline FC 30 [14] & 0,55 \\
\hline A 50 [14] & 0,50 \\
\hline P 50 [14] & 1,08 \\
\hline P 25 [14] & 0,88 \\
\hline P 25 FC 30 [14] & 1,07 \\
\hline P 25 FPP [14] & 0,83 \\
\hline V 50 [14] & 1,30 \\
\hline V 25 [14] & 1,48 \\
\hline CLM0\% [15] & 0,81 \\
\hline CLM25\% [15] & 0,78 \\
\hline CLM50\% [15] & 0,69 \\
\hline CLM100\% [15] & 0,60 \\
\hline
\end{tabular}


Observando os valores encontrados de coeficiente de capilaridade para as argamassas estudadas nesse artigo fica fácil observar uma tendência linear de crescimento do coeficiente conforme se aumente o teor de argila incorporada. Exceção é feita ao traço contendo $87 \%$ de incorporação de argila, que corresponde ao traço com $1 \mathrm{~mol}$ de cal hidratada reagindo com 2 mols de argila, o que sugere que o balanço estequiométrico teórico ocorre em torno dessas proporções de mol.

Conforme esperado previamente, os menores valores de coeficiente de capilaridade ocorrem no traço A0\% que não contém presença de argila e os maiores ocorrem no traço $\mathrm{A} 100 \%$, que contém apenas com a presença desta matéria. Fica comprovado assim que de fato os problemas patológicos destacados em [4] e [5] ocorrem em decorrência do uso de argilas nas argamassas e que a substituição desse material por cal hidratada ameniza tal problema. Conforme já descrito, o traço A87\% comprova também que é possível encontrar uma quantidade de mols de cal hidratada que reagiram com determina quantidade de mols de argila e amenizaram os problemas de cargas elétricas livres presente nas argamassas, evitando desta maneira que surjam problemas patológicos nos revestimentos. Essa proporção de mols está bem próxima de $1 \mathrm{~mol}$ de cal hidratada para 2 mols de argila teórica.

Destaca-se também que todos os traços estudados (A0\%, A62\%, A76\%, $\mathrm{A} 87 \%$, A100\%) presentes na tabela 4 apresentaram valores de coeficientes de capilaridade aceitáveis se comparados com as argamassas estudadas por autores brasileiros $[13,19]$ presentes na tabela 5 e por autores internacionais $[14,15]$ presentes na tabela 6, o que evidencia a possibilidade do uso de argamassas contendo cimento, areia e argila balanceada com cal hidratada, conforme usava-se nas obras históricas destacadas em [1-3].

\section{CONCLUSÕES}

Desta maneira conclui-se que o balanço estequiométrico, ainda que teórico, da cal hidratada com a argila, fornece bons parâmetros para evitar patologias que porventura existiriam com o uso de argamassas com apenas argilas. Por se tratar de um material muito barato em relação à aglomerante cal, a argila não pode ser ignorada no uso de argamassas de revestimento, já que além de proporcionar boa economia ao sistema revestimento, confere excelentes parâmetros de trabalhabilidade a esse material de construção. Destaca-se também a motivação histórica para o uso de material argiloso, já que se sabe que diversas obras ao longo da história mundial, que ainda se encontra em vida útil, foram executadas com argamassas contendo argilas, em épocas que a tecnologia e o estudo de argamassas eram muito mais precários [1-3].

Por fim destaca-se que o traço teórico com 1 mol de cal hidratada e 2 mols de argila, pode ser empregado para argamassas de revestimento, uma vez que os valores de coeficiente de capilaridade obtidos se mostram adequados se comparados com outros autores, e que portanto a absorção de água por capilaridade não seria uma problema para esta argamassa destaca.

\section{REFERÊNCIAS}

1 Carvalho JDN. Sobre as origens e desenvolvimento de concretos reforçados. Revista Tecnológiva. 2008;17:19-28. 
2 Meinheit D, Felder AL. Vintage steel reinforcement in concrete structures : a comprehensive and invaluable treatise on all forms of steel reinforcement employed in the design and construction of reinforced concrete of long ago; 2014.

3 Pedroso FL. Concreto: as origens e a evolução do material construtivo mais usado pelo homem. Revista Concreto. 2009;53:14-19.

4 Oliveira RA, Rego WA, Ferreira SEM, Mota JMF. Manifestações Patológicas em Revestimentos de Argamassas com Saibros. X Congreso Latinoamericano de Patología y XII Congreso de Calidad en la Construcción. 2009.

5 Carasek H. Patologia das Argamassas de Revestimento. Em: Livro Materiais de Construção Civil. Instituto Brasileiro de Concreto. 2014.

6 Newhouse EL. The Builders. Washington, D.C.: The National Geographic Society; 1992.

7 Ministero dei beni e dele attività culturali e del turismo. Colosseo di Roma [acesso em 02/08/2017]. Disponível em: http://www.beniculturali.it.

8 Silva NG, Campiteli VC, Gleize PJP. Argamassa de revestimento de cimento, cal e areia de brigatem de rocha calcária. IV Simpósio Brasileiro de Tecnologia das Argamassas. 2011.

9 Mattana AJ. Medeiros MHF. Silva NG. Costa MRMM. Analytic hierarchy process to chosse between natural aggregate and crushed rock sand for producing coating mortar. Revista Ambiente Construído. 2012;12(4):63-79.

10 Quarcioni VA, Zuquim MPS, Menezes PML, Chostoli FF, Oliveira MCB. Caracterização microestrutural de argamassas selecionadas de cimento e cales hidratadas cálcica e dolomítica por microscopia óptica. IV Simpósio Brasileiro de Tecnologia das Argamassas. 2011.

11 Silva AP, Silva BM, Carneiro AMP. Estudo de argamassa de cimento, cal hidratada e areia natural compostas como resíduo de lodo proveniente das estações de tratamento de água - ETA's. I Simpósio Brasileiro de Tecnologia das Argamassas. 2005.

12 Lagazzo A, Vicini S, Cattaneo C, Boter R. Effect of fatty acid soap on microstructure lime-cement mortar. Construction and Building Materials. 2016;116:384-390.

13 Stolz CM, Masuero AB. Analysis of main parameters affecting substrate/mortar contact area through tridimensional laser scanner. Journal of Colloid and Interface Science. 2015;455:16-23.

14 Palomar I, Barluenga G, Puentes J. Lime-cement mortars for coating with improved thermal and acoustic performance. Construction and Building Materials. 2015;75:306314.

15 Samiei RR, Daniotti B, Pelosato R, Dotelli G. Properties of cement-lime mortars vs. cement mortars containing recycled concrete aggregates. Construction and Building Materials. 2015;84:84-94.

16 ABNT NBR 13276. Argamassa para assentamento e revestimento de paredes e tetos Preparo da mistura e determinação do índice de consistência. 2016.

17 ABNT NBR 13279. Argamassa para assentamento e revestimento de paredes e tetos Determinação da resistência à tração na flexão e à compressão. 2005.

18 ABNT NBR 15259. Argamassa para assentamento e revestimento de paredes e tetos Determinação da absorção de água por capilaridade e do coeficiente de capilaridade. 2005.

19 Bauer E, Salomão MC, Filho HR. Avaliação de argamassas industrializadas quanto à capilaridade e retração de acordo com os critérios da NBR 13281 e do método MERUC/CSTB. XI Simpósio Brasileiro de Tecnologia das Argamassas. 2015

20 UNE-EN 1015-18. Methods of test for mortar for masonry. Part 18: Determination of water absorption coefficient due to capillary action of hardened mortar. Associação Espanhola de Normalização e Certificação (AENOR). 2003. 